\title{
Breaking down hybrids
}

A general explanation has been found for the puzzling performance of $\mathrm{F}_{1}$ hybrids, which can have increased vigour but also reduced fertility compared with their parents. A study in snapdragons suggests that these opposing features can arise from the interaction of genome-wide expression noise that is phenotypically silent in the parent species.

Variation in gene expression across species is widespread, but linking this variation to its phenotypic consequences requires a trait that is well characterized genetically and morphologically — one example of this is the dorsoventral asymmetry of the Antirrhinum flower. The authors examined expression variation in two of four transcription-factor genes that control this conserved trait. They then correlated the variation in the expression of these two genes (CYCLOIDEA (CYC) and RADIALIS $(R A D))$ with the degree of flower asymmetry in $\mathrm{F}_{1}$ hybrids.

First, the phenotypes and expression levels of Antirrhinum majus $C Y C$ and RAD mutants were used to generate a GEM (gene expressionmorphology) map. Here, genotypes are quantitatively related, through their expression levels, to variation in flower ventralization. When wild-type $R A D$ and $C Y C$ alleles of various other Antirrhinum species were characterized for their expression levels and positioned on the GEM, they mapped to a plateau, which means that the variation in gene expression that exists within each species did not affect the phenotype. However, crosses between species would be expected to tip the genotypes off this plateau: indeed, when double-heterozygote hybrids were generated by crossing Antirrhinum species that have divergent $C Y C$ or $R A D$ expression levels, lower expressing hybrids yielded more ventralized flowers (the figure shows a fully ventralized flower).

A visualization tool similar to the GEM map was used to model how this cryptic expression variation relates to variation in fitness between parental and hybrid strains. In this 'gene expression-fitness' map, there is a zone surrounding the fitness optimum, or peak, in which variation in gene expression values is largely neutral. The extent of this neutral zone, and its shape, will depend on the size of the population and the selective forces acting on the loci. The drifting away of one gene from the optimum would be of little fitness consequence to the population, but the drift of, say, 1,000 genes would impose substantial fitness costs.

What then is the explanation for hybrid superiority and inferiority? Two species will occupy different spaces around the fitness peak. When those two species are crossed the amount of expression noise of each species is partly cancelled out, as the variance in gene expression in the hybrid is lower than in the parents. This cancelling out brings the fitness of the hybrid closer to the

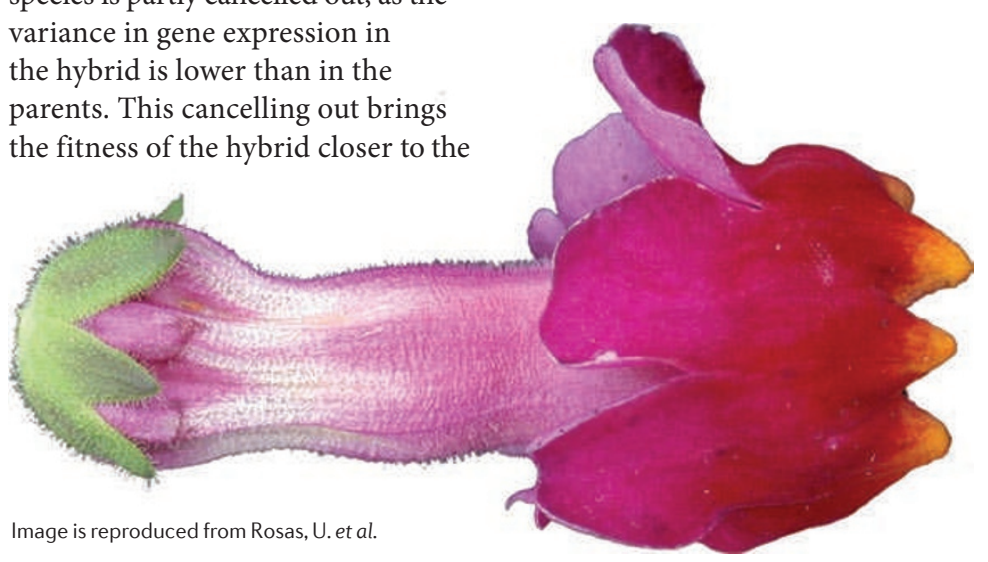

fitness optimum. From the $\mathrm{F}_{2}$ generation onwards, however, this advantage breaks down owing to Mendelian segregation. This model applies to genes involved in conserved traits, such as physiology or growth, and so accounts for the hybrid vigour seen in $\mathrm{F}_{1}$ but not subsequent generations. As mentioned above, however, the shape of the neutral zone depends on the particular locus: for genes that have more complex interactions, such as those involved in fertility, the shape of the neutral zone may make the $\mathrm{F}_{1}$ generation fall outside the neutral zone. This scenario might therefore contribute to the sterility of hybrids, for example.

Existing models to account for the properties of hybrids - such as heterozygote advantage - have several weaknesses, so a statistical explanation based on genotype-fitness maps for multiple loci has broad appeal and can be tested in other systems.

Tanita Casci

ORIGINAL RESEARCH PAPER Rosas, U. et al. Cryptic variation between species and the basis of hybrid performance. PLoS Biol. 8, e1000429 (2010) 